\title{
Innovating approaches for scientific education and research in environmental and marine sciences
}

\author{
Erika Mioni*, Silvia Merlino** \\ * Istituto Comprensivo Statale n²- Complesso “2 Giugno”, Viale A. Ferrari, 19121 La Spezi, Italy \\ ** Istituto di Scienze Marine del CNR, Forte Santa Teresa, 19032 Pozzuolo di Lerici, La Spezia, Italy
}

\begin{abstract}
Citizen-science can give a valid help to scientific investigation, and this is especially true for environment monitoring programs. However, motivate and enable volunteers in a long-term commitment to a scientific problem represents an essential problem. The presented project is an example on how this long-term commitment can be guaranteed trough an educational path that involves students, not only in data collection but also in problem definition and data analysis. We tried to identify and harmonizing the existing educational plans for improving STEM teaching and learning (STEM is a curriculum for educate students in Science, Technology, Engineering and Mathematics in an interdisciplinary and applied approach, integrating the four disciplines into a cohesive learning path based on real-world applications), prioritizing hands-on learning to increase student interest and engagement. In particular, we describe an example of how two different approaches for educate students on marine sciences can interact in the context of environmental monitoring, applied to problems such as biodiversity, pollution from marine litters, and the the impact of this problem on the marine environment.
\end{abstract}

Keywords: Environment; marin-litter; citizenscience; peer-education; non-formal-education; biocenosis, inter-generational education.

\section{Introduction}

Now day, more and more often students already possess cultural heritage in many discipline, thanks to the easy access to information (internet, media, television, etc.). Nevertheless (or perhaps precisely because of this!) a key role is played by education, to avoid a mere accumulation of information not controlled and even referenced, and of short-living concepts, but, instead, identify and develop, in students, the necessary skills and competences that will help them to "participate in an effective and constructive way in social and working life in an increasingly diverse society"1. Especially for scientific discipline, an innovative pedagogical approach is necessary, to overcome traditional science teaching made of huge amount of concepts, often not related with the real world, and to build a critical but conscious attitude towards technological innovation, scientific research and environmental issues, that will encourage the development of free and motivated personalities able to make personal choices. It is only through a constant exercise on situations drawn from reality, that we can build an appropriate scientific attitude. Moved by such considerations, teachers of Istituto Comprensivo ISA 2 of La Spezia, in collaboration with researcher of ISMAR-CNR Institute, decided to involve students of secondary school in projects concerning monitoring programs in marine field, sharing methodologies and practices developed in two different contexts, i.e. BluePaths project and Seacleaner project, in conveying non-formal education, research and environmental awareness. Both are projects born, independently, with the intention to take into account both science education and scientific investigation. The former monitor biocenosis of beaches and sublittoral zone, analysing littoral flora and fauna from upper and middle shore, using

\footnotetext{
${ }^{1}$ Recommendation of the European Parliament and of the Council (2006/0962/EC) on Key Competences for Lifelong Learning ,
} published in the Official Journal of the European Union on 30 December 2006/L394. 
and integrating the Reef-Check Protocol ${ }^{2}$ to the Ligurian and North Thyrrenian sea, since these areas are subject to intensive boat traffic, and biological invasions of new species, a daily and common treat for coastal communities that is often underestimated; the latter is devoted to estimate the quantity, typology, accumulation rates of marine litter, i.e. "number of items (or weight or volume) per unit surface of beach per unit of time" of beached trash, for the most part deposited ashore from the sea. This is one of the negative consequence of our "plastic age", and the survey is performed following and adapting the UNEP/IOC (Cheshire 2009) protocol for marine litter and the $\mathrm{MSFD}^{3}$, as described in next sections.

Both projects provide data collected during repeated monitoring campaigns in the same place and at a distance of months, to allow to deduce information on the type and rate of accumulation of materials (the biological one and the anthropogenic one), estimate the possible origin and even make assumptions about the prevalence of marine currents and wave motion in the area under study. It result clear that a problem for visual survey of the littoral is that data collection of marine litter requires long time and the work of a lot of people. The need of long-term data series, to increase collected information, can be satisfied by the citizen-science, i.e. the active participation of volunteers in both kind of scientific projects (Cerrano et al. 2013, Newman et al., 2012). On the other hand, motivating and enabling all these volunteers in a long-term commitment to a scientific problem can represent a problem. For this reason, in 2013 BluePaths and SeaCleaner decided to collaborate in an unique project, sharing resources, methods, means and objectives, with the idea that the requested long-term commitment can be ensured trough a comprehensive educational path that involves students, not only in data collection but also in problem definition and data analysis. This has been possible, specially for high school students, thank to an innovative tool in higher education and vocational training: the "work-related learning”4, with the Italian Legislative Decree n. 77 of April 15, 2005 (Article 4). In our particular case, we used this tool to design an experience that is, in our opinion, particularly profitable as it presents vantages in the education of the students, as well as in research; the final product is also twofold: an educational experience which is highly motivating for students, and a scientific and technological "output" with a sound scientific and also social value.

BluePaths and SeaCleaner are actually still cooperating and operating in sampling sites of the coastal Tuscan and Ligurian $\mathrm{MPA}^{5}$. The selected areal is of great importance because it surrounds the Pelagos Santuary of marine mammals, animals at particular risk for micro-plastic pollution. From the scientific point of view, so, merging data of the two projects will represent the starting point necessary to proceed in trying to evaluate the effect that fragmentation of marine litter can have on coastal ecosystem (filter feeders such as bryozoans and other benthonic indicators) and evaluate the impact on the food chain.

\footnotetext{
2 Protocols for the Coastal Environmental Monitoring (CEM) along Italian coasts ( www.reefcheckitalia.it) partner of the world wide Reef Check Foundation (www.reefcheck.org))

3 MSFD-Marine Strategy Framework Directive: Guidance on Monitoring of Marine Litter in European Seas, Reference Report by the Joint Research Centre of the European Commission., 2013.

4 The work-related learning is a different way of connecting the school with work, through courses designed, implemented and evaluated under the responsibility of schools and on the basis of appropriate agreements with public and private companies, chambers of commerce, etc., available to accommodate students for periods of learning in the work situation.

5 Marine Protected Areas of 5 parks: National Park of the Tuscan Archipelago and of Cinque Terre, Regional Natural Park of Migliarino-San Rossore-Massaciuccoli, of Montemarcello-Magra and of Portovenere.
} 
Characteristic of both projects is to have an educative moment that extends outside the school and becomes an opportunity for students to practice theoretical skills acquired at school in their family environment. Moreover, both projects are harmonizing the existing educational plan, by adding hands-on learning activities to increase student interest and engagement in the STEM fields.

\section{Marine litter problem and biodiversity loss: two starting point for motivate students.}

The Anthropogenic Marine Debris (AMD), or marine litter problem, is a current global problem: it damages fisheries, environment, tourism, and can represent a threat to human health. AMD includes any processed solid material discarded or abandoned that ends up in the marine environment, for the most part of plastic material that, upon fragmentation, is transformed into particles easily assimilated in the food chain. Waste' introduction progressively increases with the gradual urbanization of the coasts, and the effect is even more enhanced for 'closed' basins such as the Mediterranean. Studies show the establishment of zones of accumulation also in the Mediterranean Sea (Suaria and Aliani, 2014), even if in the central and western part of this sea the rate of accumulation of floating AMD up now is not so well known. Thus, we are not able to evaluate the severe consequences that macro and micro litter could have on marine and coastal communities (Fossi et al., 2014), especially those belonging to the valuable biological areas such as the "Pelagos Sanctuary" (shelter of marine mammals in the Nord part of Mediterranean) and MPA. Recently, the European Community has been included marine litter amongst the 11 descriptors of MSFD, incorporating 56 indicators of Good Environmental Status (Galgani et al., 2013), soliciting researchers to fill the gap existing in the presently available data: especially for the coasts surrounding the Pelagos Sanctuary in Mediterranean Sea, information are still very scarce (http://www.strategiamarina.isprambiente.it/sintesirisultati-consultazione-2014). Moreover no study has been carried on to establish clear relationships between litter density and possible effects on benthic communities, and studies are lacking which provide extensive beach survey data in Mediterranean area and a clear picture of the trends in the medium/long-term, with possible biodiversity loss consequences. These environment problems are often unknown by students, and not treated in standard scholastic programs. For better motivate pupils we decided, so, to involve a group of high schools students not only in the monitoring actions, but also in the phase of "problem solving": for marine litter surveys, their task was create a protocol useful and friendly, able to be applied by a large range of people, and design the informatics architecture of an application for Android device, easy-to-use but methodologically correct (the app SeaCleaner). We tried to lead students to broaden their perception of the problem quantifying it, observing with their eyes, counting and classifying the enormous amount of anthropogenic waste that is dumped into the sea every day, analyzing the possible main sources of waste drained into the sea and how it interacts with the biosphere. Strictly linked to this topic, in fact, the one of the loss of biodiversity is a problem that involves more and more natural environments, and it is interesting to study the possible correlation with the crescent pollution from non-biodegradable waste of our coasts. For this reason, through the BluePath project a group of secondary school students have been trained on marine biology and monitoring techniques, with scholastic and extra scholastic paths, in order to be able to realize, under supervision of teachers and experts, visual census of beached and underwater bio-indicators belonging to benthonic communities. Biological monitoring activities has started in 2014 and are continuing 
until today, focusing on two areas in particular: the reserve of San Rossore and the island of Pianosa, in the park of the Tuscan Archipelago (Fig.2).

\section{Methods}

\section{Participation in science activities: a teaching strategy for the consolidation of skills.}

SeaCleaner design: during firsts step of the project (2013), students involved in the ideation and construction of the app SeaCleaner collected information about the marine litter problem and about existing standard protocols for monitoring it. After that, they have been divided into two groups, depending on their scholastic training. Task of first group was to adapt existing material to our specific purposes, i.e. to design a table of data collection for macro-litter [longer $>2,5 \mathrm{~cm}$ ] that could be used also by volunteers but it was at the same time exhaustive and rigorous, and it was adapted to be inserted in an informatics device. The second group' task was to transfer this table in an electronic format, and develop the utilities necessary for the acquisition and recording of data in the device, implementing the application for the Android operating system. Both groups were involved in the final phase: to test the effectiveness of the app and any shortcomings of structural design. The use of a similar device in data acquisition campaign is very fruitful, as very important is the standardization of the acquired data in order to allow the comparison of different campaigns of study, performed in different places and times. Without a common standard, data are not consistent, especially for the purpose of environmental planning, that need careful information about the amount of macro-waste streams beached or floating (UNEP 2012). For this reason, students involved in the protocol design have payed attention to use protocol available in literature, and to adapt it to their purposes analyzing the situation of the places where they live. The app still have different aspects to be completed, as the graphical interface: this affects its use and public spread, and for this reason it is still under test. Nevertheless, the protocol SeaCleaner it is available; it has been used by students during the second part of the project, in 2014, to acquire information on the distribution and fragmentation of marine-litter in different areas of the monitored areas, in parallel to the acquisition of information on biological communities (biocenosis) of different marine organism (benthos) in the same distribution areas, essential in order to investigate possible correlations between the state of health of ecosystems studied and the abundance of litter found (Project BluePaths).
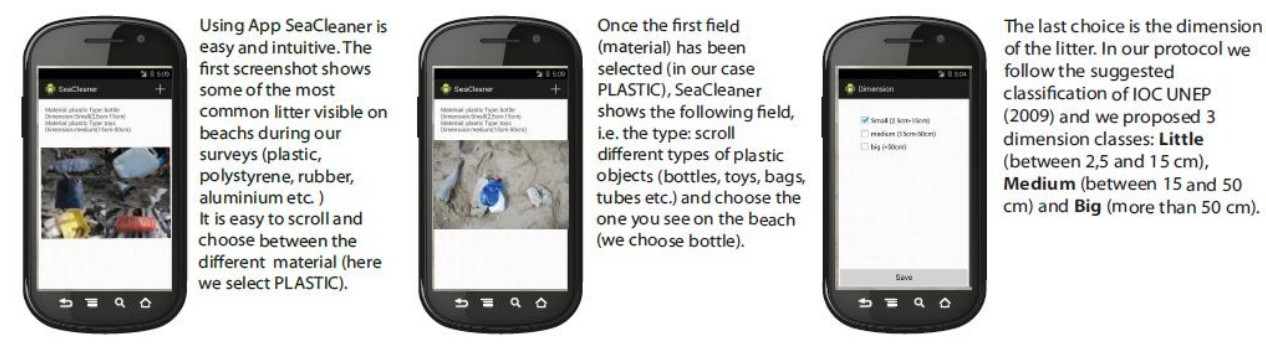

Figure 1. Application SeaCleaner, developed with the aim to be extremely "user-friendly following Guidelines of UNEP/IOC-“Quick poll” case (Cheshire et al. 2009). Added value is identified in the ease of acquisition, management and storage of data directly into electronic format and stored on Remote Server. The final layout is not yet ready, but here we can show how it would appear once completed in all the graphical part. 
BluePaths, i.e. education and training in marine monitoring activities: starting from the environmental potential that the natural area surrounding La Spezia itself offers, the project tries to stimulate the observation skills and intellectual curiosity, encouraging the development of rational assumptions and disseminating the basic principles of experimental scientific method. We are aware that, in the age of secondary school, the possibility to acquire skills in marine biology allow students to apply them directly in surveys of benthic species, in the supralittoral, interditial, upper infralittoral coastal areas. Students are involved in the study of biodiversity, and a technical language is used but, above all, our hope is to trigger a conscious learning of the experimental scientific method and its application to the monitoring techniques for coastal emerged and submerged zones. Principal arguments of the training phase are awareness of marine ecological interactions, classification of marine living or beached/dead organisms and classification of beached, floating marine natural and anthropogenic litter, techniques of coastal monitoring. Students are pushed to build their own sampling instruments (quadrats of $50 \mathrm{~cm} \mathrm{x} 50 \mathrm{~cm}$ ), and tools (manual, checklists etc.); as they become experts during this training period, they have to perform tasks of tutoring towards smaller students, peers or adults (volunteers or students' families) that get trained and are and involved in activities of Citizen Science in the framework of the project. This phase of peer-education and inter-generational education is very important, especially for students of the age between 11-14 years. This approach has proved very useful to increase the self-esteem and sense of responsibility of students, and greatly improve their academic performance as well as social behaviour. After data collection, students learn basic techniques of statistical data processing and produce their own table and graphs. This scientific training is also an occasion for orientating students towards Upper Secondary Schools. The threeyear training educational path can be so summarized:

- 1st year: basic training in marine biology, acquisition techniques for autonomy in monitoring emerged coast; acquisition of operating methods for visual census in submerged coast, peer learning: "how to be part of a Research Team”; Tutoring role with families; active participation in Citizen Science.

- 2nd year: knowledge' enhancement in marine biology, start autonomous monitoring; active participation of Citizen Science; "how to remain in a Research Team”.

- 3rd year: upgrading of skills, selection of Peer educators, assigning tutoring roles in Research campaigns, participation in research campaigns; active participation of Citizen Science; permanence in the research team; active engagement in dissemination activities, peer education and Citizen Science.

\section{Results and Discussion}

SeaCleaner involves 7 students of high school in 2013, and 17 students of high school and 20 of middle school in 2014. In 2015 is active with 40 students coming from different high schools of Liguria, Tuscany and Emilia Romagna regions, and 40 students of middle school of La Spezia province. Participation of students from different schools and degrees resulted in highly motivating experiences especially for the younger people, and may be effective in contrasting the school dropout, mainly perceived in the range of age between 14 and 17 years. Moreover, students were involved in real research operation, working with scientists, educators and environment operators: from the set up of the SeaCleaner App, to the beach 
monitoring and the following classification and analysis of collected items and preparation of tables and graphic; These approach have proven their value in order to stimulate the students and pupils to the topics covered and the methods used (Locritani et al., 2013, Merlino et al., 2014).

In the same way, Blue Paths have involved, in scholastic period 2013-2014, 34 classes of secondary school students, networking 3 Scholastic institutes (one high school and two middle school) and, moreover, 100 family (parents of the students) in citizen-science activities. In the same period (May 2014), 6 students were engaged in scientific activities of visual survey (up and under-water) in the Pianosa Island, working with researcher of ISMAR Institute. During scholastic year 2014/2015 the number of networking Institute has grown (with the adhesion of another high school of La Spezia and one of the town of Ancona, on Adriatic Sea). In addition to the involvement of students (35 classes) and families (100) in the monitoring activities conducted mainly in the San Rossore Park, a research internship was carried out in the Island of Pianosa (September 2014, participants 11 students of secondary schools, two teachers and two researchers) and a third internship is foreseen for May 2015, with the participation of 13 secondary school students, one university students and four researchers/teachers).

For both projects, participation and co-working of students coming from different institutes/regions, has been essential for fostering social skills and intra-and intergenerational communication (peer education), a result that is giving essential clues to researchers and teachers - for improving and evaluating, in the future, this kind of informal teaching methods.
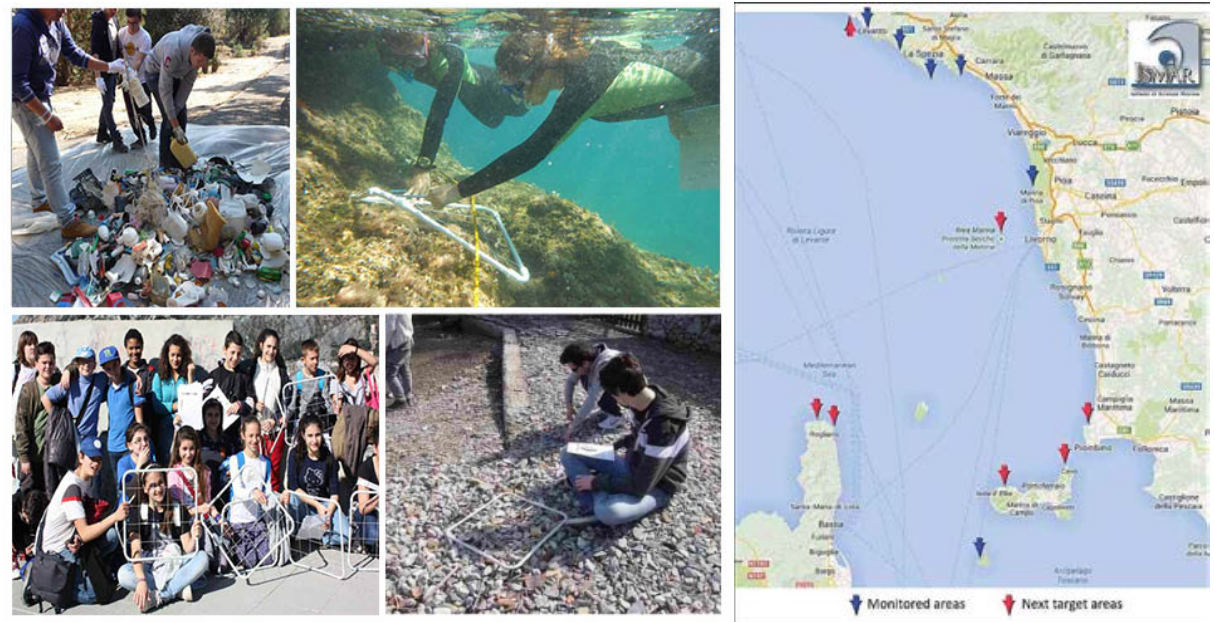

Figure 2a. Monitoring the beaches where marine litter is deposited by currents get feedback on the quantities and types of waste in our sea. Collected data will be compared with biological information investigated, in the same areas, with PVC quadrates on beach and underwater, by high and middle school students. 2b. BluePath/SeaCleaner are monitoring 6 coastal protected areas (blue arrows), surrounding a part of Mediterraneo included in the Pelagos Santuary, thanks to a network of research organizations and university, schools, marine parks and volunteers. Red arrows are next target areas.

Scientific results of this cooperation are indeed interesting, as presentation of preliminary results during European and International Conferences ${ }^{6}$; collection of

\footnotetext{
${ }^{6}$ EGU, European Geological Union, Wien, 17-21 April 2013; Congress "Ecology for a sustainable blue and green growth, Ancona, 16-18 Settembre 2013; IORC International Conference, Barcelona, 17-21 november 2014
} 
important awards and grants $^{7}$; preliminary indication about litter distribution and check list of benthic fauna and flora of studied areas (Merlino et al. 2014); but other outcomes, social and educational, shared by both projects, should be pointed out:

- hands-on experiences are successful in influencing individual behaviour for preventing and reducing, as possible, marine pollution and for perceiving the importance of environment conservation.

- synergy among very different partners (parks, researchers, local authorities, citizens, environmental centres, teachers and students) represent an effective pushpull impulse for maintaining a long lasting engagement in scientific research.

- activities carried out in parks are effective for increasing the interest and importance of these areas and to consider tangible actions aimed at preserving the environment.

- the involvement of many students and families in survey/educational/awareness activities is one of the most important output of the project, raising to spontaneous "peer education" and “inter-generational education”.

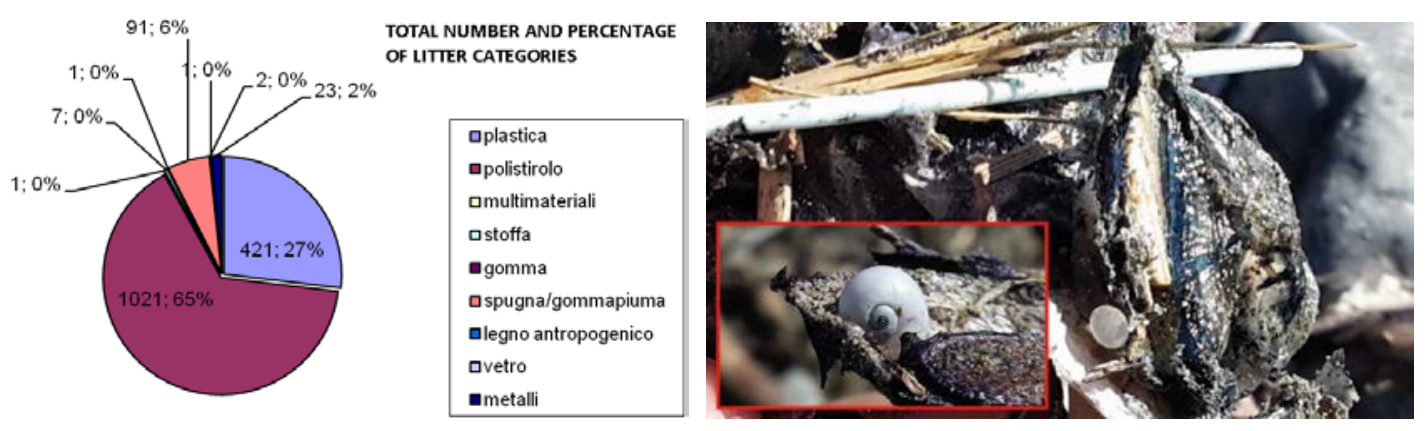

Figure 3. Examples of project's output realized by students: in the photo the bio-indicator "Janthina Palida”, frequent in Pianosa island and, in March 2015, found in Palmaria island; it is often associated with "floating rafts" formed by "Velella Velella” and polystyrene fragment/plastic pellet. The graphic shows the abundance of litter in Palmaria in the same period (March 2015): polystyrene and plastic prevail.

\section{Conclusions and further improvements}

Cooperation between the projects BluePaths and SeaCleaner show a clear example of interdisciplinary methodology in both didactic approach and scientific research; it has led students to discover little-known aspects of matters just mentioned in class, to deepen their knowledge of some aspects of the world of environmental research and computer science. The shared project not only has grown for number of students and schools, but also, now, include the membership of the Liguria District of Marine Technology, the Department of Biology of the University of Pisa, the National Institute of Geophysics and Volcanology, and MPA in Liguria and Tuscany, in order to continue in next years, with the enlargement of the network of involved organizations and schools, as well as of the areal under study. Different acquisition and analysis methods will be used (high definition photography, microscope observation) and a GIS system for data storage, maps and information will be implemented. The increasing number of adhesion of students and Parks implies a grown, day after day, of collected data: our next scientific goal would be the statistical analysis of correlations between parameters emerged from SeaCleaner survey

\footnotetext{
${ }^{7}$ Special International Award Ramoge “Alain Vatricain” 2012, EU Days for Scientific dissemination and Best-practice of workrelated learning in 1st National Convention on work-related learning, ‘Salone dell’Orientamento’ of Genoa, November 2013.
} 
(accumulation rates, fragmentation index, distributions of materials' type in different beaches etc.) and biological data on biocenosis and benthic communities provided by BluePaths survey.

As concern the educational/social goals, we are satisfied by the growing interest of the scholastic institutions towards our project. Our first goal it is not to form good scientists, but to form good citizens, and we think we are doing everything that we can to pursue it

\section{References}

UNEP, United Nations Program for Environment (2012). State of the Mediterranean Marine and Coastal Environment 2012. Highlights for Policy Makers UNEP Mediterranean Action Plan (MAP). Barcelona Convention, Athens, 2012.

Suaria G. \& Aliani S. (2014). Floating debris in the Mediterranean Sea. Marine Pollution Bulletin. 86(1-2): 494-504.

Locritani M., Furia S., Giacomazzi F., Merlino S., Mori A., Nacini F., Nardi E., Stroobant M., Talamoni R., and Zocco O. (2013). La Spezia and the research network for outreach and education in marine sciences. In: Proceedings of European Geosciences Union General Assembly. Wien: EGU 2013, April 7-12.

Fossi M.C., Coppola D., Baini M., Giannetti M., Guerranti C., Marsili L., Panti C., de Sabata E., Clò S. (2014). Large filter feeding marine organisms as indicators of microplastic in the pelagic environment: The case studies of the Mediterranean basking shark (Cetorhinus maximus) and fin whale (Balaenoptera physalus). Marine environmental research. 100: 17-24

Galgani F., Hanke G., Werner S., Oosterbaan L., Nilsson P., Fleet D., Kinsey S., Thompson R.C., van Franeker J., Vlachogianni T., Scoullos M., Mira Veiga J., Palatinus A., MatiddiM., Maes T., Korpiunen S., Budziak A. Leslie H., Gago J and Liebezeir G. (2013). Guidance on Monitoring of Marine Litter in European Seas 2013. JRC Scientific and Policy reports, European Commission.

Cheshire A.C., Adler E., Barbière J., Cohen Y., Evans S., Jarayabhand, S., Jeftic L., Jung R.T., Kinsey S., Kusui E.T., Lavine I., Manyara P., Oosterbaan L., Pereira M.A., Sheavly S., Tkalin, A., Varadarajan S., Wenneker B., Westphalen G. (2009). UNEP/IOC Guidelines on Survey and Monitoring of Marine Litter. UNEP Regional Seas Reports and Studies, No. 186; IOC Technical Series.

Cerrano C, Milanese M, Mioni E, Palma M, Pantaleo U, Previati M, Rossi G, Scinto A, Turicchia E, Ponti M. (2013). Reef Check Italia onlus: a network to improve civil participation in marine environment assessment. In: Riassunti del XXIII Congresso della Società Italiana di Ecologia (S.It.E). pp 16-18. Ancona, 16-18 September.

Merlino S., Stroobant M., Locritani M., Talamoni R., Furia S., Muccini F., Abbate M., Nacini F., Mori A. e C. Carmisciano. (2014). ALLA SCOPERTA dei TESORI del MARE: Scienza e Tecnologia, Memoria Popolare e Identità Culturale di una città della costa ligure. Miscellanea INGV. (22): 21 pp.

Newman, G., Wiggins, A., Crall, A., Graham, E., Newman, S., Crowston, K. (2012). The future of citizen science: emerging technologies and shifting paradigms. Frontiers in Ecology and Environment. 10(6): 298-304. 